\title{
Radiolucent lesion identified in unerupted mandibular left first permanent molar: Case report and literature review
}

\author{
Aki Yamana, Kazuhiko Nakano, Hidekazu Sasaki, Rena Okawa, \\ Kazuyo Fujita, Michiyo Matsumoto and Takashi Ooshima \\ Department of Pediatric Dentistry, Osaka University Graduate School of Dentistry \\ 1-8 Yamada-oka, Suita, Osaka 565-0871, JAPAN
}

\begin{abstract}
Intracoronal resorption in an unerupted developing tooth is rare and most lesions of this type are incidentally found in radiographs taken for other purposes. A 5Y10M-old girl was referred to our clinic by a general practitioner for detailed examination of radiolucency identified in the unerupted mandibular left first permanent molar. Intraoral examination revealed that the affected tooth was unerupted, while the radiographic findings demonstrated a wide radiolucent area in the coronal part of the affected molar, which extended close to the pulp. There were no signs or symptoms associated with the tooth at the first examination. At 6Y4M of age, the affected tooth had emerged into the oral cavity, though the patient reported no symptoms. We performed surgical removal of the gingival tissue corresponding to the occlusal surface of the tooth under infiltration anesthesia and macroscopic appearance showed the affected tooth to be intact. However, when the enamel of the affected tooth was removed, a hollow portion approximately $2 \mathrm{~mm}$ into the tooth with no pulp exposure was revealed. Calcium hydroxide was applied to the bottom of the cavity and restoration with cement was performed. Three months after treatment, the patient had no abnormal signs or symptoms. A literature search found 15 similar cases, in which mandibular dentition was more frequently affected than maxillary dentition. As for treatment modalities in those cases, conservative restoration was preferably selected, though extraction was inevitable in approximately half.
\end{abstract}

\author{
Key words \\ First permanent molar, \\ Radiolucent area, \\ Unerupted tooth
}

\section{Introduction}

Intracoronal resorption in an unerupted developing tooth, also known as pre-eruptive intracoronal radiolucency, is a rare condition, with most cases incidentally discovered in radiographs taken for other purposes ${ }^{1,2)}$. This type of lesions is generally found in a single tooth, and the typical radiological feature is a well-circumscribed radiolucent area identified in the coronal aspect of the affected tooth ${ }^{3}$. The prevalence of this lesion is reported to be approximately $6 \%$ in all subjects and $2 \%$ of teeth, and the most favorable location identified is the

Received on March 23, 2010

Accepted on June 7, 2010 mandibular permanent molar and premolar region, especially the mandibular first molar ${ }^{4}$. The lesion itself does not cause any symptoms in most cases, though it has carious-like features in radiographic findings ${ }^{5,6)}$. The etiology of this type of resorption is speculative, though it is widely accepted that a breach of reduced enamel epithelium, which forms a barrier against the surrounding environment during tooth development of the dental follicle, could allow connective tissue to directly contact the enamel ${ }^{5,7-11)}$. It is also considered that these lesions should be managed conservatively, though several case reports have noted that extraction of the affected tooth was inevitable ${ }^{8,10)}$.

This type of radiolucent lesion was first described more than 50 years $\mathrm{ago}^{7}$. However, only limited 

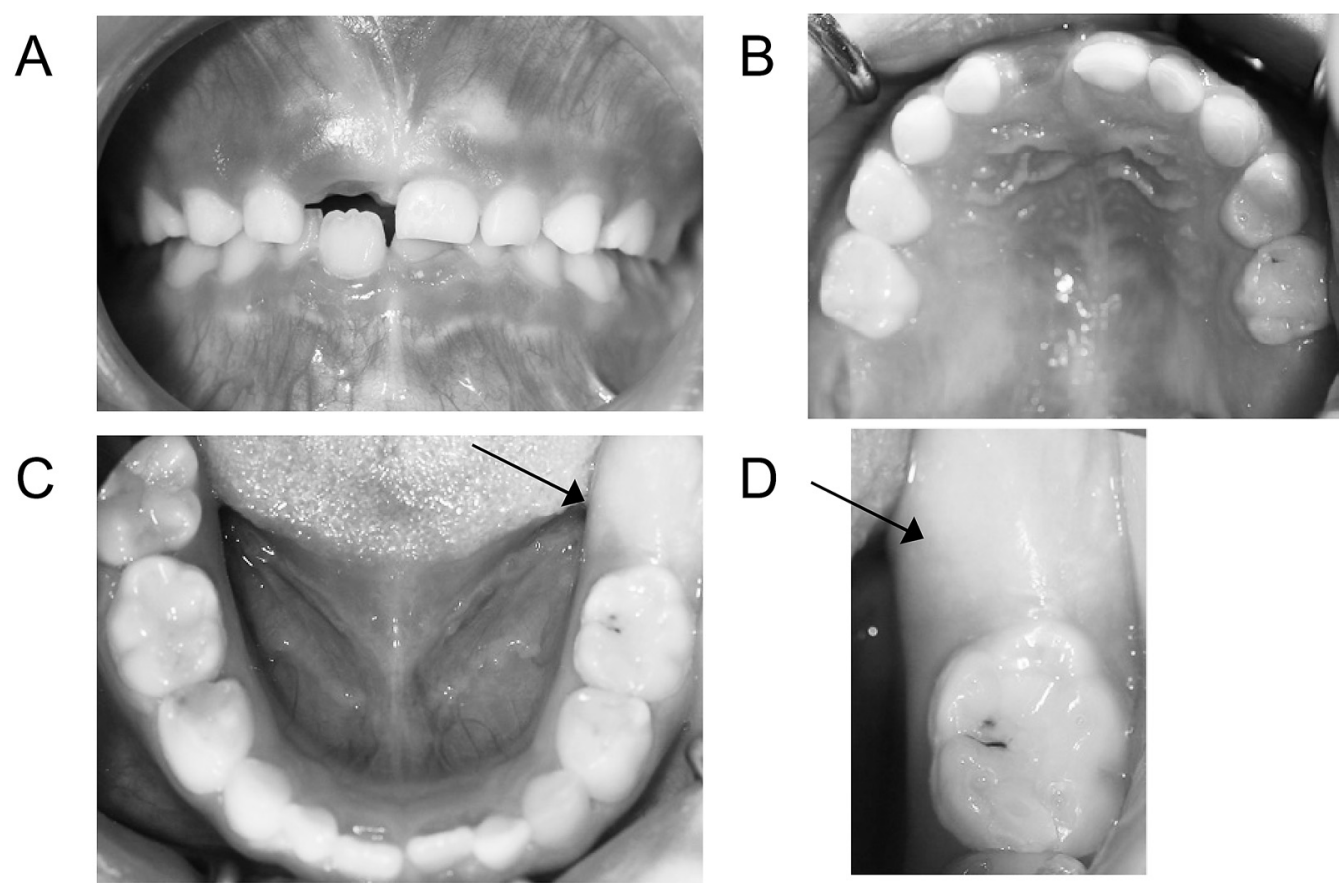

Fig. 1 Intraoral photographs taken at first examination

Shown are frontal (A), maxillary (B), and mandibular (C) views, and a magnified image of the mandibular left first molar region (D). Arrow indicates gingival tissue overlying the affected tooth.
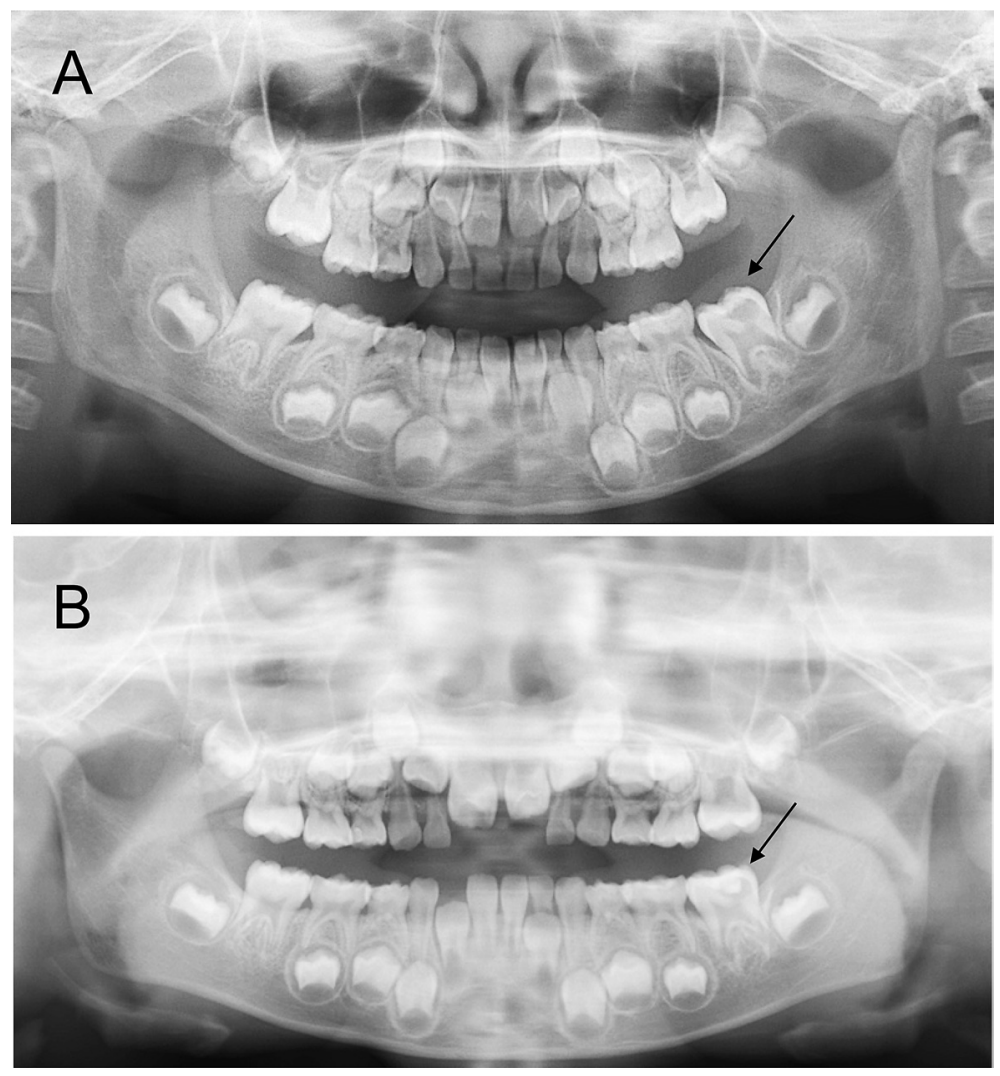

Fig. 2 Orthopantomographs taken in this case

Images were obtained at the first visit at the age of 5Y10M (A) and at 6Y4M (B). 
Table 1 Summary of this and other reported cases

\begin{tabular}{ccccccc}
\hline Case & Age & Gender & Location* & Symptoms** & Treatment & References \\
\hline 1 & 10 & M & LL7 & ND & Extraction & {$[7]$} \\
2 & 11 & M & LR4 & ND & Extraction & {$[12]$} \\
3 & 11 & F & LR7 & ND & Restoration & {$[8]$} \\
4 & 11 & M & LL7 & ND & Extraction & {$[8]$} \\
5 & 12 & M & LR7 & ND & Restoration & {$[8]$} \\
6 & 7 & M & LL6 & ND & Restoration & {$[13]$} \\
7 & 6 & F & LL6 & ND & Restoration & {$[13]$} \\
8 & 13 & M & LL3 & ND & Restoration & {$[9]$} \\
9 & 11 & F & LR7 & ND & Restoration & {$[9]$} \\
10 & 6 & M & LR6 & ND & Extraction & {$[9]$} \\
11 & 10 & F & LR5 & ND & Restoration & {$[3]$} \\
12 & 13 & M & LL7 & Intermittent dull pain & Extraction & {$[10]$} \\
13 & 11 & F & LL7 & No symptoms & Extraction & {$[11]$} \\
14 & 9 & F & LL7 & Mild intermittent pain & Extraction & {$[2]$} \\
15 & 6 & F & LL6 & No symptoms & Restoration & Present case \\
\hline
\end{tabular}

*LL7; mandibular left second molar, LR4; mandibular right first premolar, LR7; mandibular right second molar, LL6; mandibular left first molar, LL3; mandibular left canine, LR6; mandibular right first molar, LR5; mandibular right second premolar.

**ND: not described

information is available and no systematic review of case reports has been presented. Herein, we describe a case of intracoronal resorption identified in the area of the mandibular left first molar in a 5 -year-old girl and also present a review of pertinent literatures.

\section{Case Report and Review of Literature}

A 5Y10M-old girl was referred to our clinic by a general practitioner for detailed examination of a radiolucent area identified in the area of the unerupted maxillary left first permanent molar. Our intraoral examinations showed that the mandibular central incisors and mandibular right first molar were emerged into the oral cavity, whereas the mandibular left first molar had not yet emerged (Fig. 1). Orthopantomographic examinations identified an intracoronal radiolucent lesion in the area of the mandibular left first permanent molar (Fig. 2A). There were no signs or symptoms reported at the first examination. Based on these findings, we diagnosed the condition as intracoronal resorption in the unerupted mandibular left first molar and decided to wait for the affected tooth to emerge into oral cavity.

At the age of $6 \mathrm{Y} 4 \mathrm{M}$, the affected tooth had emerged into oral cavity, though the patient reported no symptoms (Fig. 2B). In addition, the surrounding soft tissues appeared to be normal and showed no evidence of inflammation. We decided to perform surgical removal of the gingival tissue corresponding to the occlusal surface of the tooth under infiltration anesthesia, after which the macroscopic appearance of the occlusal surface of the tooth seemed to be normal with an intact enamel surface. Then, removal of the enamel layer was performed beginning with the distal fossa and a hollow area approximately $2 \mathrm{~mm}$ into the tooth were encountered. No pulp exposure was seen, and filling with calcium hydroxide and cement restoration were performed. Three months after that treatment, the patient had no abnormal signs or symptoms in the area of the affected tooth. After confirmation of prognosis, we intend to replace the cement with composite resin in the near future.

Cases of intracoronal resorption in an unerupted developing tooth as well as those of pre-eruptive intracoronal radiolucency, were collected from a database search (Table 1) ) $^{2,3,7-13}$. The ages of the reported patients ranged from 6 to 13 years old, with no gender distinction found. All affected teeth were part of the mandibular dentition, among which second permanent molars were the most frequent, 
followed by first molars. In addition, lesions were also identified in premolar region and one case report described a lesion detected in a canine tooth. There were no signs or symptoms reported in most cases, while intermittent pain was reported in two. As for treatment modalities utilized, conservative restoration with or without pulp capping was preferably selected, though extraction was inevitable in approximately half of the cases.

\section{Discussion}

A pericoronal radiolucent lesion in an unerupted tooth is regarded as an uncommon clinical finding, with most cases generally recovered incidentally in radiographs taken for other purposes. The present case was identified in an orthopantomograph obtained for treatment planning by a general practitioner, who considered that the radiolucent findings might represent dental caries, though the affected tooth was unerupted. Although there were no signs or symptoms reported, the general practitioner decided to refer the patient to our clinic for consultation regarding treatment planning for the tooth. When we noted the radiographic appearance of the affected tooth, we recalled cases with an unerupted primary molar treated with restoration materials that had been incidentally found in radiographic examinations. As for the etiology of this type of lesion, it is generally known that ankylosed teeth that received previous restoration treatment become submerged and behave as if they were unerupted. However, there was no evidence that the present affected tooth had ever erupted, including reports from the general dentist and the guardians of the patient. Thus, we eliminated that possibility and made a diagnosis of a pericoronal radiolucent lesion in an unerupted tooth.

An epidemiological survey held in Australia analyzed approximately 2,000 children aged from approximately 3-12 years, and reported that the occurrence rate of intracoronal resorption was approximately $6 \%$ of the subjects and $2 \%$ of the teeth examined ${ }^{4)}$. It is of interest that these lesions are reported to be identified in the mandibular dentition 6.4 times more frequently than in maxillary dentition, though the reasons remain unclear. In addition, approximately $45 \%$ of the cases reported were the mandibular first molar, followed by mandibular first premolar (18\%) and mandibular second molar (17\%), while the mesial part of the tooth was found to be affected more frequently as compared to the central and distal parts. In the present case, an intracoronal radiolucent area was identified in the distal part of the first molar, which is regarded as a common location, but in a less frequent part of the tooth. On the other hand, our review of other case reports showed that the mandibular second molar was affected more frequently than the first molar. We speculate that there might be differences in these distributions among ethnic groups, which should be investigated in future studies. Since early detection is advantageous for conserving the affected tooth, it is important to keep in mind the preferable location for this type of lesion, especially when performing radiographic examinations for other purposes.

The radiolucency of this type of lesion was generally limited to less than one-third of the dentin thickness in approximately $85 \%$ of the cases reported, with radiolucency covering from one- to two-thirds in $12 \%{ }^{4}$. In the present case, radiolucent area extended close to the pulp cavity, thus we regard it as an uncommon and severe case. We applied calcium hydroxide to the bottom of the cavity close to the pulp and restoration with cement was performed. It has been suggested that dentin adjacent to the pulp might be more resistant to the resorptive process as compared to other dentinal regions ${ }^{14}$. In the present case, there were no signs or symptoms reported for up to three months after the procedure. Nevertheless, we plan to perform longitudinal periodical examinations in the future. In previously reported cases, extraction was selected for approximately half, though conservative treatment is regarded as superior. It has also been proposed that the affected tooth is possibly susceptible to severe dental caries due to a lack of dentine tissue after eruption, while the radiolucent area was found to be enlarged in some cases, even though the tooth was unerupted ${ }^{2)}$. Therefore, early detection and timely intervention are important for cases of intracoronal resorption in an unerupted developing tooth.

It would be informative to have histopathological findings available from cases of intracoronal resorption in an unerupted developing tooth. Several studies have analyzed the histopathological features of extracted teeth, in which vital fibroblasts and collagen, as well as a moderate number of blood vessels and few ossification foci were found ${ }^{8}$. A separate study reported no pathological changes in pulp tissue, in which the roof of the pulp chamber was intact and non-inflamed pulp with areas of extensive resorption and repair along the remaining 
coronal portion of the tooth were also identified ${ }^{9)}$. It has been speculated that a breach of reduced enamel epithelium, which forms a barrier against the surrounding environment during tooth development, could allow connective tissue to directly contact the ename ${ }^{5,7-11)}$. In addition, uncommon occlusal pits were reported in a case with an intracoronal radiolucent lesion identified in an unerupted mandibular right second molar, which were thought to indicate the locations of direct contact between dentin and connective tissue ${ }^{11}$. Furthermore, chronic periapical inflammation of primary teeth may promote epithelial disruption and osteoclastic activity ${ }^{11)}$, whereas that would only be applicable for permanent teeth with predecessors.

In summary, we treated a case of intracoronal resorption in an unerupted developing tooth identified at the mandibular left first permanent molar, which was incidentally found in radiographic examinations performed for other purposes. When performing radiographic examinations, it is important to keep coronal resorption of unerupted teeth in mind as a possible finding.

\section{References}

1) Moskovitz, M. and Holan, G.: Pre-eruptive intracoronal radiolucent defect: a case of a nonprogressive lesion. J Dent Child 71: 175-178, 2004.

2) Klambani, M., Lussi, A. and Ruf, S.: Radiolucent lesion of an unerupted mandibular molar. Am J Orthod Dentofacial Orthop 127: 67-71, 2005.

3) Brooks, J.K.: Detection of intracoronal resorption in an unerupted developing premolar: report of case. $J$
Am Dent Assoc 116: 857-859, 1988.

4) Seow, W.K., Lu, P.C. and McAllan, L.H.: Prevalence of pre-eruptive intracoronal dentin defects from panoramic radiographs. Pediatr Dent 21: 332-339, 1999.

5) Skaff, D.M. and Dilzell, W.W.: Lesions resembling caries in unerupted teeth. Oral Surg Oral Med Oral Pathol 45: 643-646, 1978.

6) Coke, J.M. and Belangaer, G.K.: Radiographic carieslike distal-surface enamel defect in an unerupted second premolar. ASDC J Dent Child 48: 46-49, 1981.

7) Blackwood, H.J.: Resorption of enamel and dentine in the unerupted tooth. Oral Surg Oral Med Oral Pathol 11: 79-85, 1958.

8) Grundy, G.E., Pyle, R.J. and Adkins, K.F.: Intracoronal resorption of unerupted molars. Aust Dent $J$ 29: 175-179, 1984.

9) Rankow, H., Croll, T.P. and Miller, A.S.: Preeruptive idiopathic coronal resorption of permanent teeth in children. J Endod 12: 36-39, 1986.

10) Taylor, N.G., Gravely, J.F. and Hume, W.J.: Resorption of the crown of an unerupted permanent molar. Int J Paediatr Dent 1: 89-92, 1991.

11) McNamara, C.M., Foley, T., O'Sullivan, V.R., Crowley, N. and McConnell, R.J.: External resorption presenting as an intracoronal radiolucent lesion in a pre-eruptive tooth. Oral Dis 3: 199-201, 1997.

12) Wooden, E.E. and Kuftinec, M.M.: Decay of unerupted premolar. Oral Surg Oral Med Oral Pathol 38: 491-492, 1974.

13) Wood, P.F. and Crozier, D.S.: Radiolucent lesions resembling caries in the dentine of permanent teeth. A report of sixteen cases. Aust Dent J 30: 169-173, 1985.

14) Yaacob, H.B.: The resistant dentine shell of teeth suffering from idiopathic external resorption. Aust Dent J 25: 73-75, 1980. 